\title{
IOT SENSOR NETWORK BASED APPROACH FOR AGRICULTURAL FIELD MONITORING AND CONTROL
}

\author{
Shruti A Jaishetty ${ }^{1}$, Rekha Patil ${ }^{2}$ \\ ${ }^{1}$ M.Tech (4th SEM) Student, Department of Computer Science and Engineering, Poojya Doddappa Appa College of \\ Engineering, Kalaburgi, Karnataka, India \\ ${ }^{2}$ Associate Professor, Department of Computer Science and Engineering, Poojya Doddappa Appa College of \\ Engineering, Kalaburgi, Karnataka, India
}

\begin{abstract}
Modernization of the farming process is one of the crucial steps for a country like India, which has to import tremendous amount of grains and agro products from other countries to meet the demand of 1.2 billion populations. Various architectural and technological improvements have been suggested in the past and have been widely adopted over the years to improve the productivity of the agricultural field. One of the major challenges of the agriculture is the proper monitoring of the soil health, the environment, and adjusting the irrigation as well as the plant clear according to this observation. Traditionally, Indian farmers have adopted manual observation based technique which has resulted in either excessive use of pesticides or under use of watering or many issues which results in less efficient and less productive crops. The proposed work is about having control over the irrigation and monitoring of the agricultural field using IoT. The system connects physical sensing devices with the cloud and connects the irrigation control mechanism with the cloud. This keeps an immense analysis and problem solving capability to the overall architecture. Results have shown that we can mitigate data in real time with exceedingly low latency and based on that we can take the decisions.
\end{abstract}

Keywords: Irrigation Control, Agricultural Field Monitoring.

\section{INTRODUCTION}

Internet of things (IoT), is a cloud of interconnected physical devices, which can communicate with each other over the Internet. Physical devices such as microcontrollers, microprocessors, actuators, and sensors will not directly communicate with the Internet; they do so by using an IoT gateway. This entire infrastructure is known as IoT infrastructure. For example we can take a Home Lighting System, where all the switches are been connected to the main controller which is connected to the internet. The smart farm, embedded with IoT systems, could be called a connected farm, which can support a wide range of devices from diverse agricultural device manufacturers. Also, connected farms could provide more intelligent agriculture services based on shared expert knowledge.

Improvement of agriculture field has become biggest challenging for the countries like India, so new technologies have to be adopted. We have implemented a novel methodology of physical parameter monitoring, data display, data integration to the cloud, alert generation and predicting the future values with the help of MATLAB analysis. We have used temperature sensor, rainfall sensor, light sensor and the moisture sensor. These sensors have been installed in the agriculture field to collect the data, and thus data is mitigated into the cloud with the help of IoT hub(Thingspeak).So user can have a real time data visualization, with the help of MATLAB analysis user can predict the future parameter values. By predicting the moisture value user can have control over the agriculture field by using a MQTT, by sending commands. MQTT is a machine to machine communication protocol which is based on pub-sub service. In this we have subscribed to the servers by creating channels, server could be "iot.eclipse.org" and "test.mosquitto.org". The remote monitoring solution that we offer can be monitored in real time through any remote devices including mobiles or tablets. This provides the flexible for the data visualization, data understanding, and the predictive analysis also given the scope for the farmers to prepare for the advanced data which might appear in the future.

The organization of this work is as follows. Section 2 contains the related work, section 3 is about the proposed work, and section 4 covers the implementation and result analysis. Finally conclusion and future work has been discussed in the section 5 .

\section{RELATED WORK}

Monitoring of soil moisture and groundwater levels using ultrasonic waves to predict slope failures, [1] used an ultrasonic waves to predict the slope failures when there is a heavy rainfall, and they have used a method of monitoring of soil moisture. Optimal sensor placement strategy for environmental monitoring using Wireless Sensor Networks, [2] has used wireless sensor networks to determine the optimal sensor placement method for the monitoring of environmental changes. They have also been used a geostatistical analysis and Monte Carlo theory to develop the strategy. 
The realization of precision agriculture monitoring system based on wireless sensor network, [3] has used wireless sensor networks to design the monitoring of agriculture, at the same time the system is based on the real time monitoring of agriculture environmental information such as temperature, humidity and the light intensity. Precision agriculture monitoring framework based on WSN, [4] proposes that WSNs play a vital role in decision making, monitoring of agricultural field and optimization of agricultural resources. WSNs also help to know the real time data related to the agricultural field and the condition of the crop, so the farmers can make sure that they are ready to face the future conditions related to their agricultural field. .

Development of Knowledge Sharing System for Agriculture Application, [5] proposes a methodology where the data related to the agricultural field can be shared using the Knowledge sharing system. The main theme of the research is to design a methodology to share the data and it also involves inheriting of agricultural data. The methodology involves data collection by two methods. First one is the automatic environmental data collection by a sensor, and second method is the experienced data collection by a farmer. Optimal design of solar powered fuzzy control irrigation system for cultivation of green vegetable plants in Rural India, [6] proposes methodology called fuzzy-logic that acts as a solution for irrigation control in order to cultivate the vegetable plants. Smart Farming System Using Sensors for Agricultural Task Automation, [7] proposes a unique methodology that links smart sensing system and smart irrigator system which is collectively known as smart farming process.

\section{PROPOSED WORK}

We propose a Novel IoT sensor network for monitoring the environmental parameter in an agriculture field. Mitigating this data to the cloud through a secured IoT hub (Thingspeak), and then run and predictive analysis and the machine learning on the gathered data (mat lab deployed at Thingspeak).The proposed system overcome the limitations of traditional GPRS based system through protocols like MQTT, secured HTTP, which not only ensured that the data is safe and secured, but the entire communication is over an authorized secured socket layer which gives immense security to the data.The proposed system also have bandwidth compatible and cross level compatible, therefore the client level solution can be run either on the mobile, PC or on the tablet. The remote monitoring solution that we offer can be monitored in real time through any remote devices including mobiles or tablets. This provides the flexible for the data visualization, data understanding, and the predictive analysis also given the scope for the farmers to prepare for the advanced data which might appear in the future.

The proposed system is $4 \mathrm{G}$ compatible, it is not limited by the bandwidth and high latency, which is experienced for the GPRS based system. At the same time the MQTT protocol itself free, the overall operating cost for controlling mechanism is null in comparison with GSM based system, where each SMS's has a cost which is determined by the tariff of the service provider.

The system architecture is composed of sensors (temperature, moisture, rainfall and the light), which are installed in the agriculture field. These sensors will be collecting the environmental parameters. The sensed data is mitigated into the cloud through an IoT gateway (thingspeak); thingspeak gives a real time data visualization.

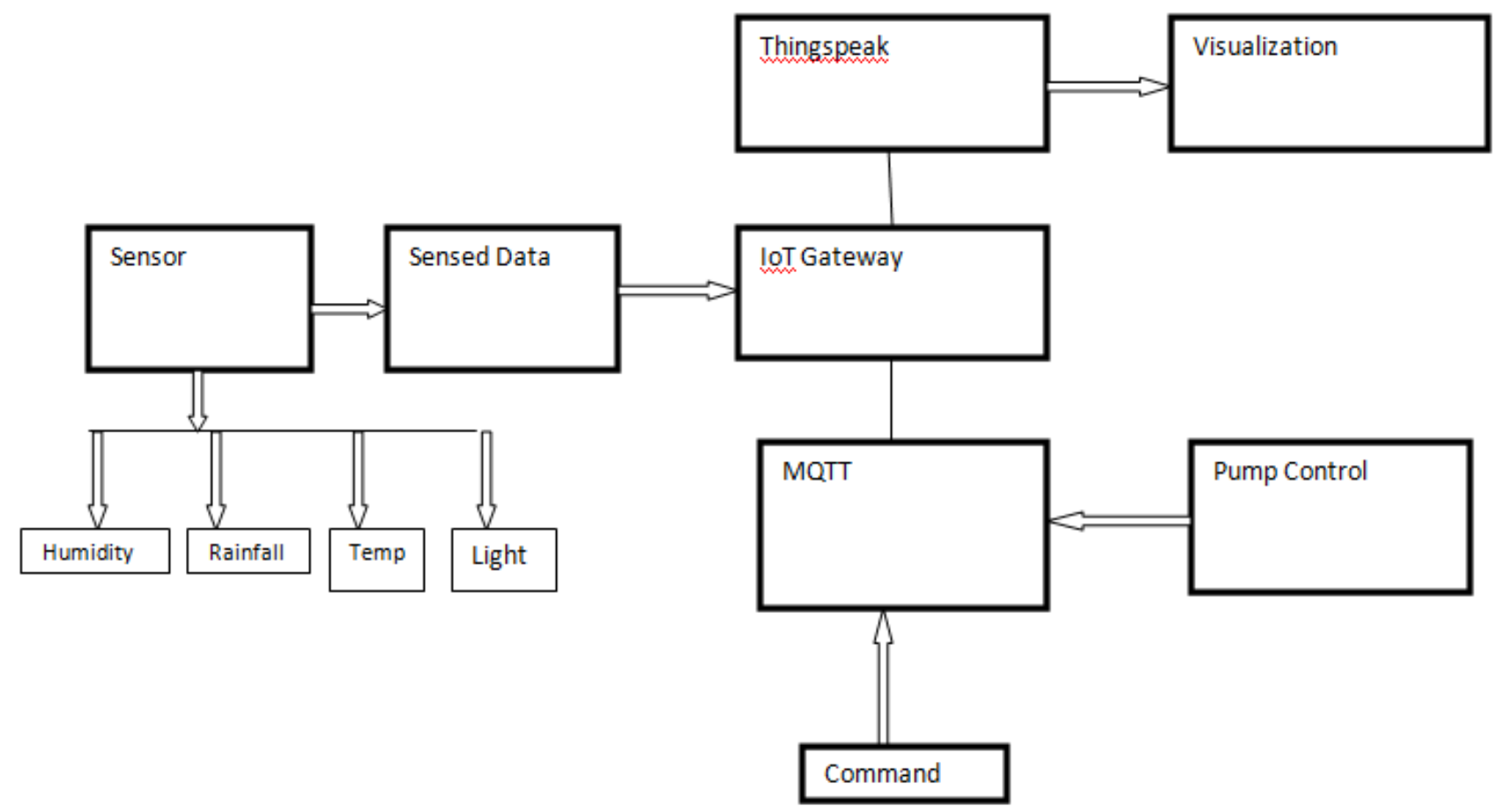

Figure.1 System Architecture 
We are using a light weight communication protocol MQTT, by using whichwe are having control over the irrigation in the form of controlling the relay. In the MQTT protocol the user has to subscribe to the server "iot.eclipse.org" or "test.mosquitto.org", and at the same time the user has to connect to the "rupam/Agro". The commands are passing through MQTT protocol to control the agriculture field.

\section{IMPLEMENTATION AND RESULT ANAYSIS}

User can analyze the output in the thingspeak, which will give the graphical notations of all the values. Thingspeak is an internet of things application and is an open source. ThingSpeak can also acts as an application programming interface in order to store and retrieve the data using the HTTP protocol over the internet or via a Local area. We can see the graphs of temperature value, light value, soil moisture value and the rainfall value.

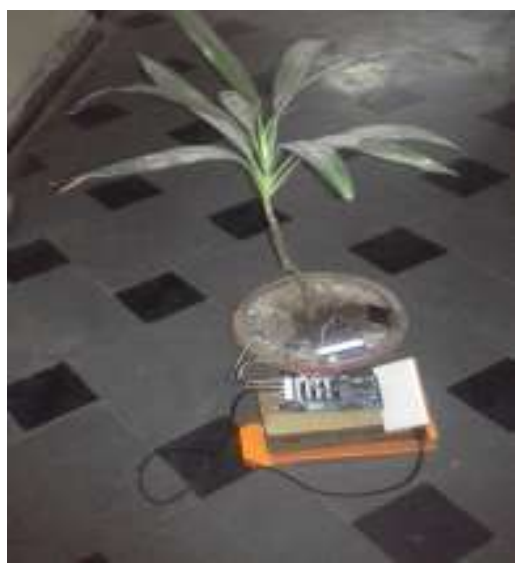

Fig-2: Experimental Setup

A real time data visualization can be analyzed in the thing speak, which is an IOT hub. The following figure shows the real time data visualization.

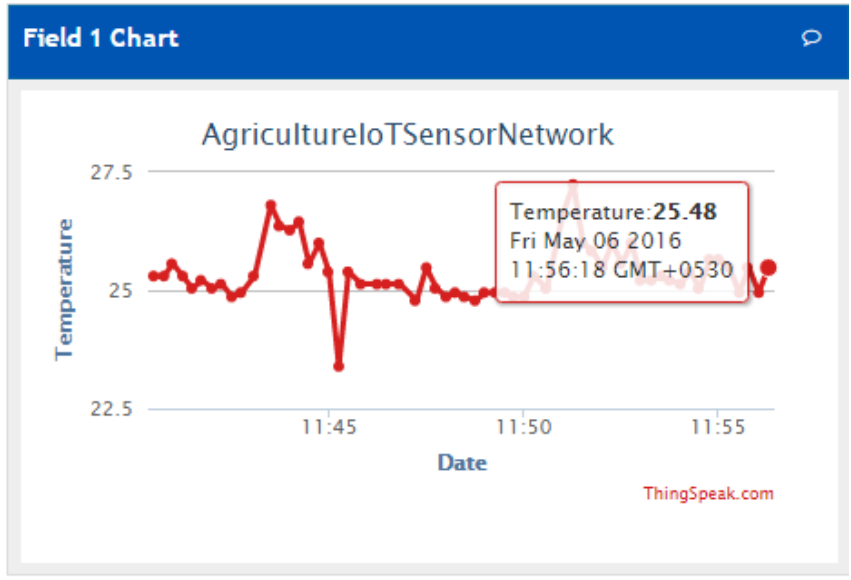

Chart-1: Analysis of Temperature value

The graph in the above figure suggests that the temperature value is analyzed in the thingspeak IoT hub. The graph is plotted with temperature value $\mathrm{v} / \mathrm{s}$ date.

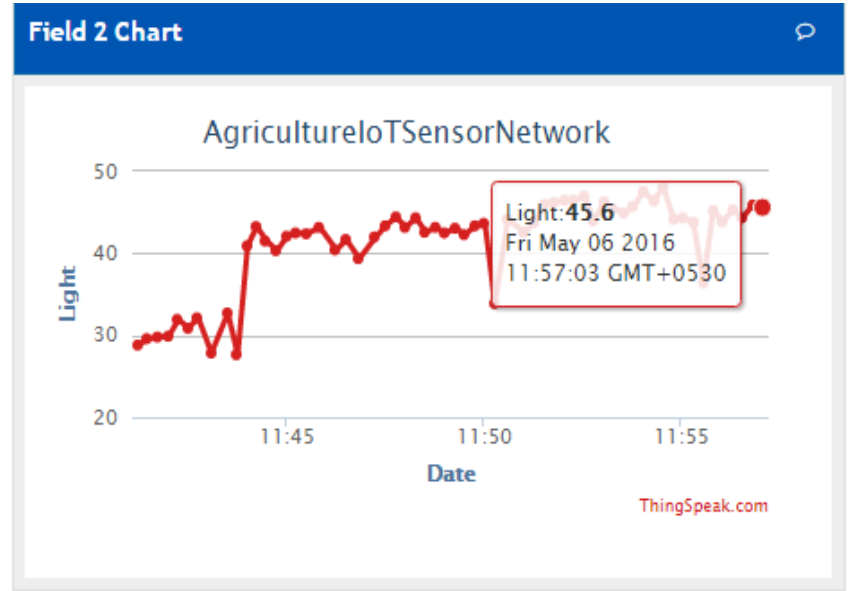

Chart-2: Analysis of light value

The above graph shows that light value is changed with change in the sensor.

The below figure shows the graph of moisture value. The value is being analyzed by taking vaious reading of the soil value

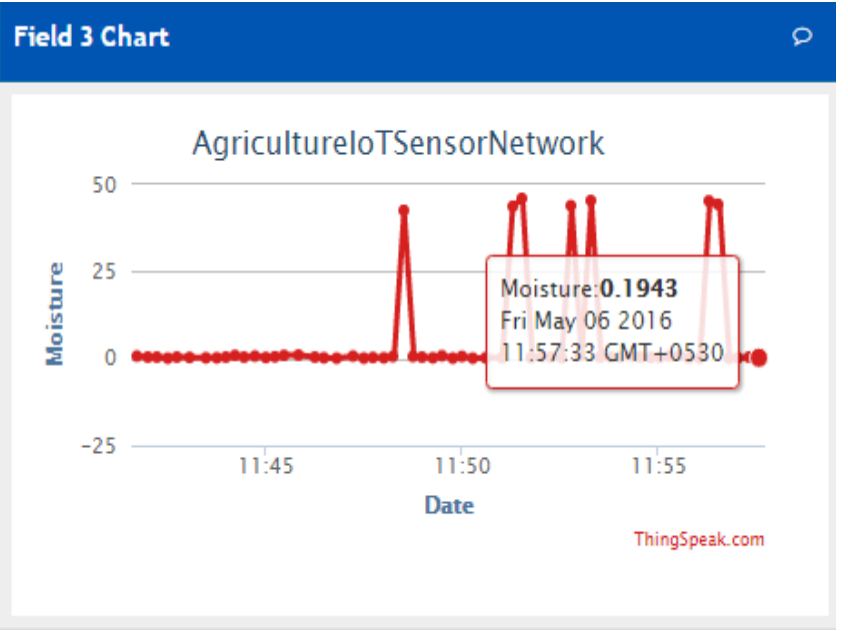

Chart-3: Analysis of Moisture value

The below figure shows that the rainfall value is analyzed in the thingspeak, by dissipating its value into the cloud.

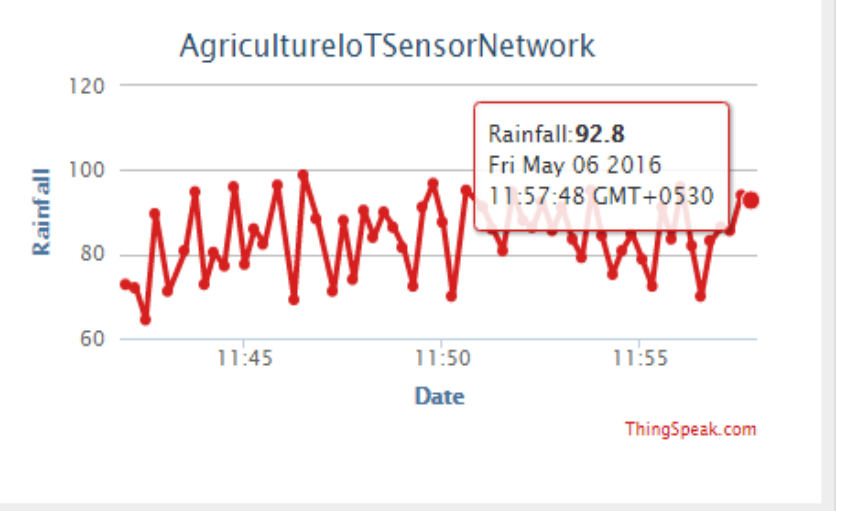

Chart-4: Analysis of Rainfall value 


\section{CONCLUSION AND FUTURE WORK}

Improvement of the crop productivity is a major challenge in the countries like India, the technological improvement is a mandatory work to improve the crop productivity to support and sustain the need for ever green population of our country. In the past several sensor driven network have been proposed to successfully monitor the large agriculture field. However most of the technology does not offer on the data mining technique and predictive analysis, which limits the data usage to accurate state of the field and crop. We propose a novel technology by means of which gathered data from physical sensing devices is mitigated in the cloud, where a machine learning technique could in real time produce not only the alerts corresponding to the current state of the environment and the crop but at the same time can offer predictive analysis of the future state of environment as well as crops.

The IoT based architecture also offers real time realization and analysis of data which can be used across the globe in conjunction with the parameter been monitored through other parts of the world to understand the abnormal behavior of the similar kind of the crop. Our result shows that the proposed system has a very optimal latency for controlling the system as well as high packet delivery rates and accuracy for mitigating the data. The system can further been improved by incorporating new self learning techniques which could deployed in the cloud to understand the behavior of the sensing data and can take autonomous decisions.

\section{REFERENCES}

[1]. Migdall, S.; Klug, P.; Denis, A; Bach, H., "The additional value of hyperspectral data for smart farming," Geoscience and Remote Sensing Symposium (IGARSS), 2012 IEEE International , vol., no.,pp.7329,7332, 22-27 July 2012

[2]. Jhuria, M.; Kumar, A; Borse, R., "Image processing for smart farming: Detection of disease and fruit grading," Image Information Processing (ICIIP), 2013 IEEE Second International Conference on , vol., no.,pp.521,526, 9-11 Dec. 2013

[3]. Qiang Wang, Terzis A. and Szalay A., "A novel soil measuring wireless sensor network," Instrumentation and Measurement Technology Conference (I2MTC), 2010 IEEE, vol., no., pp.412,415, 3-6 May 2010

[4]. Castello C.C., Fan J., Davari A. and Ruei-Xi Chen, "Optimal sensor placement strategy for environmental monitoring using Wireless Sensor Networks," System Theory (SSST), 2010 42nd Southeastern Symposium on , vol., no., pp.275,279, 7-9 March 2010

[5]. Tanaka, K.; Suda, T.; Hirai, K.; Sako, K.; Fuakgawa, R.; Shimamura, M.; Togari, A, "Monitoring of soil moisture and groundwater levels using ultrasonic waves to predict slope failures," Sensors, 2009 IEEE ,vol., no., pp.617,620, 25-28 Oct. 2009

[6]. Lei Xiao; Lejiang Guo, "The realization of precision agriculture monitoring system based on wireless sensor network," Computer and Communication Technologies in Agriculture Engineering (CCTAE),2010

International Conference On , vol.3, no., pp.89,92, 1213 June 2010

[7]. Jiber, Y.; Harroud, H.; Karmouch, A, "Precision agriculture monitoring framework based on WSN," Wireless Communications and Mobile Computing Conference (IWCMC), 2011 7th International, vol., no.pp.2015, 2020, 4-8 July 2011

[8]. Eric D. Hunt., ET al.2008. "The development and evaluation of a soil moisture index." Int. J. Climatol. Published online in Wiley InterScience. www.interscience.wiley.com

[9]. Uchinuno, T.; Yasunaga, Y.; Keiichi, M.; Sugimoto, N.; Aoqui, S.-I, "Development of Knowledge Sharing System for Agriculture Application," Advanced Applied Informatics (IIAIAAI), 2013 IIAI International Conference on, vol., no., pp.108, 111, Aug. 31 2013Sept.4 2013

[10].Wei Lin, "Real time monitoring of electrocardiogram through IEEE802.15.4 network," Emerging Technologies for a Smarter World (CEWIT), 2011 8th International Conference \& Expo on , vol., no.,pp.1,6, 2-3 Nov. 2011

[11].Zhenyu Liao; Sheng Dai; Chong Shen, "Precision agriculture monitoring system based on wireless sensor networks," Wireless Communications and Applications (ICWCA 2012), IET International Conference on ,vol., no., pp.1,5, 8-10 Oct. 2012

[12].Singh, S.N.; Jha, R.; Nandwana, M.K., "Optimal design of solar powered fuzzy control irrigation system for cultivation of green vegetable plants in Rural India," Recent Advances in Information Technology (RAIT), 2012 1st International Conference on , vol., no., pp.877,882, 15-17 March 2012 